\title{
Eye Gel in Single-dose Container
}

National Cancer Institute

\section{Source}

National Cancer Institute. Eye Gel in Single-dose Container. NCI Thesaurus. Code C149510.

Medicinal product consisting of an eye gel presented in a single-dose container. 